\title{
Association between cardiorespiratory fitness and metabolic risk factors in a population with mild to severe obesity
}

Kathy Do ${ }^{1}$, Ruth E. Brown', Sean Wharton ${ }^{1,2}$, Chris I. Ardern ${ }^{1}$ and Jennifer L. Kuk ${ }^{1 *}$

\begin{abstract}
Background: Previous literature suggests the beneficial effects of fitness on abdominal obesity may be attenuated in obesity and abolished in severe obesity. It is unclear whether the beneficial association between fitness and health is similarly present in those with mild and severe obesity.

Methods: Patients from the Wharton Medical Clinic $(n=853)$ completed a clinical examination and maximal treadmill test. Patients were categorized into fit and unfit based on age- and sex-categories and body mass index (BMI) class (mild: $\leq 34.9 \mathrm{~kg} / \mathrm{m}^{2}$, moderate: $35-39.9 \mathrm{~kg} / \mathrm{m}^{2}$ or severe obesity: $\geq 40 \mathrm{~kg} / \mathrm{m}^{2}$ ).

Results: Within the sample, $41 \%$ of participants with mild obesity had high fitness whereas only $25 \%$ and $11 \%$ of the participants with moderate and severe obesity, respectively, had high fitness. BMI category was independently associated with most of the metabolic risk factors, while fitness was only independently associated with systolic blood pressure and triglycerides $(P<0.05)$. The prevalent relative risk for pre-clinical hypertension, hypertriglyceridemia and hypoalphalipoproteinemia and pre-diabetes was only elevated in the unfit moderate and severe obesity groups $(P<0.05)$, and fitness groups were only significantly different in their relative risk for prevalent preclinical hypertension within the severe obesity group $(p=0.03)$. High fitness was associated with smaller waist circumferences, with differences between high and low fitness being larger in those with severe obesity than mild obesity (Men: $P=0.06$, Women: $P=0.0005$ ).
\end{abstract}

Conclusions: Thus, in contrast to previous observations, the favourable associations of having high fitness and health may be similar if not augmented in individuals with severe compared to mild obesity.

Keywords: Cardiorespiratory fitness, Metabolic risk factors, Obesity

\section{Background}

The fit-fat paradox has been the topic of investigation for many years [1-5]. Specifically, it is suggested that individuals with mild obesity and a high fitness may not present with the typically expected negative health factors associated with obesity and may have lower risk of mortality than their normal-weight unfit counterparts [1-5]. The health benefits of having a high fitness are thought to be in part due to reduced visceral obesity for a given body mass index (BMI) [6]. However, the decreased abdominal obesity associated with a high

\footnotetext{
* Correspondence: jennkuk@yorku.ca

'School of Kinesiology and Health Science, York University, School of

Kinesiology and Health Science, Toronto M3J 1P3, Canada

Full list of author information is available at the end of the article
}

fitness may be attenuated with higher levels of obesity, and it is suggested that there may no differences in abdominal obesity by fitness in individuals with moderate and severe obesity [7]. Conversely, the potential beneficial associations between high fitness and health may be larger in severe obesity than milder obesity groups $[6,8]$, perhaps due to the greater deterioration in health commonly observed in severe obesity classes. Populations with moderate to severe obesity have increased in prevalence and have reached over $5 \%$ in Canada [9] but remain a relatively understudied population. Thus, the primary objective of this study is to determine the relationships between fitness and metabolic risk factors in individuals with higher levels of obesity. 


\section{Methods}

\section{Participants}

The sample included 853 patients with obesity who attended the Wharton Medical Clinic. Participants were included if they underwent measurements of blood pressure, general blood work and a standardized treadmill test during their first 3 months of enrolling at the weight management centre. All participants provided written informed consent knowing their decision to participate would not alter the care provided and that they could withdraw consent at any time. York University Institutional Review Board approved the study protocol used (Certificates: 2013-123 and \#e2017-166). The datasets generated and analyzed during the current study are not publicly available due to privacy laws associated with medical data but are available with a data sharing agreement as approved by the relevant institutional ethics committee and the health information custodian (S. Wharton).

\section{Clinical examination}

Blood samples were obtained via venipuncture after at least an $8 \mathrm{~h}$ fast to assess triglycerides, glucose, and high density lipoprotein (HDL) cholesterol using standard procedures by certified medical laboratories. Blood pressure was measured manually at the clinic by trained technicians. Preclinical hypertension was defined as blood pressure $\geq 130 / 85 \mathrm{mmHg}$ or use of hypertensive medications. Preclinical hypertriglyceridemia was defined as triglyceride $>1.7 \mathrm{mM}$ or use of lipid medications. Preclinical hypoalphalipoproteinemia was defined as HDL levels less than $1.0 \mathrm{mM}$ in men or $1.3 \mathrm{mM}$ in women or use of lipid medications. Prediabetes was defined as glucose $\geq 5.6 \mathrm{mM}$ or use of diabetes medications. Waist circumference was measured at the midpoint between the superior iliac spine and lowest rib [10]. BMI was used to categorize obesity levels: mild: BMI 30-24.9 kg/m², moderate: BMI 35-39.9 kg/m ${ }^{2}$ and severe: $\mathrm{BMI} \geq 40 \mathrm{~kg} / \mathrm{m}^{2}$ [11].

Maximal oxygen uptake $\left(\mathrm{VO}_{2 \max }\right)$ was estimated from a graded multistage treadmill Bruce protocol. Treadmill time was used to predict $\mathrm{VO}_{2 \max }$ using sex-specific equations $[12,13]$. Participants were stratified by fitness based on standard age- and sex- specific $\mathrm{VO}_{2 \max }$ cutoffs: unfit ( $<20$ th percentile) or fit ( $\geq 20$ th percentile) [14].

\section{Statistical analysis}

ANOVA and chi-squared tests were used to determine group differences in participant characteristics. Differences in health risk between mild, moderate or severe obesity groups by fitness groups were assessed using generalized linear models with obesity class (continuous term) and fitness main effect and interaction terms adjusting for age, sex and relevant medication use (diabetes, lipid or blood pressure). When the interaction term was not significant, the model was re-run without the interaction term. When the interaction or obesity group and/or fitness main effect was observed, the least squared means were reported for the obesity-fitness groups with least squared difference post hoc tests to assess group differences.

The relative risk for prevalent preclinical risk factors for the obesity and fitness groups was assessed using main effects and interaction terms adjusting for age and sex using the method proposed by Zou [15]. When the interaction term was not significant, the model was rerun without the interaction term. When the interaction or obesity and/or fitness main effect was observed, a least squared difference post hoc was conducted to assess group differences. Analyses were performed using SAS v9.4 (SAS Institute, Cary, NC).

\section{Results}

Within the sample, $41 \%$ of participants with mild obesity were fit whereas only $25 \%$ and $11 \%$ of the participants with moderate and severe obesity, respectively, were considered fit (Table 1). Individuals with high fitness tended to be younger (47.6 vs 51.4 years), had a lower BMI (35.8 vs. $41.1 \mathrm{~kg} / \mathrm{m}^{2}$ ) and more likely to be female (84.4 vs $75.9 \%, P<0.05)$.

The mean blood pressure, glucose, triglycerides and HDL adjusted by age and sex stratified by BMI and fitness category are shown in Fig. 1. As expected, BMI category was independently associated with most of the metabolic risk factors, while fitness was independently associated with SBP and triglycerides $(P<0.05)$. There were no significant differences between fitness groups within a BMI category $(P>0.05)$. Further, there were no significant differences between obesity groups within the fit category for any of the metabolic variables $(P<0.05)$. Unfit, severe obesity groups had significantly worse metabolic profiles as compared to the fit mild obesity group $(P<0.05)$ and the unfit mild obesity group (except triglycerides, $P<0.05$ ).

Those with low fitness and higher obesity tended to have a larger waist circumference (Fig. 2). There was a significant BMI $x$ fitness interaction, indicating that the differences in waist circumference between fitness groups were greater in those with higher obesity classes in men $(P=0.06)$ and women $(P=0.0005)$. However, the difference in waist circumference between fitness groups only attained significance in the moderate (Fit versus Unfit: 112.1 versus $116.5 \mathrm{~cm}, P=0.001$ ) and severe (Fit versus Unfit: 119.6 versus $129.2 \mathrm{~cm}, P<0.0001)$ obesity groups in women.

The prevalent relative risk for pre-clinical hypertension, hypertriglyceridemia and hypoalphalipoproteinemia and pre-diabetes was only elevated in the unfit moderate and severe obesity groups as compared to fit and unfit groups with mild obesity (Fig. $3, P<0.05$ ). The only 
Table 1 Participant characteristics stratified by BMl group and fitness

\begin{tabular}{|c|c|c|c|c|c|c|}
\hline & \multicolumn{2}{|l|}{ Mild Obesity } & \multicolumn{2}{|c|}{ Moderate Obesity } & \multicolumn{2}{|l|}{ Severe Obesity } \\
\hline & Fit & Unfit & Fit & Unfit & Fit & Unfit \\
\hline N & 107 & 151 & 60 & 181 & 38 & 316 \\
\hline Age $(y)$ & $51.0(10.5)^{\text {bce }}$ & $54.8(11.0)^{\text {acef }}$ & $43.9(11.2)^{\text {abdf }}$ & $53.5(11.9)^{\mathrm{cef}}$ & $44.0(11.9)^{\mathrm{abdf}}$ & $48.5(11.4)^{b-e}$ \\
\hline Sex (\%female) & 80.4 & 75.5 & 90.0 & 77.9 & 86.8 & 75.0 \\
\hline $\mathrm{BMI}\left(\mathrm{kg} / \mathrm{m}^{2}\right)$ & $32.2(1.6)^{\mathrm{b}-f}$ & $32.5(2.0)^{c-f}$ & $37.2(1.5)^{\text {abef }}$ & $37.4(1.4)^{\text {abef }}$ & $43.7(3.2)^{a-d, f}$ & $47.4(6.6)^{a-e}$ \\
\hline $\mathrm{VO}_{2 \max }(\mathrm{ml} / \mathrm{kg} / \mathrm{min})$ & $36.6(4.1)^{\mathrm{bdf}}$ & $21.6(6.1)^{\text {ace }}$ & $35.6(1.7)^{\mathrm{bdf}}$ & $20.5(6.1)^{\text {ace }}$ & $35.6(3.1)^{\mathrm{bdf}}$ & $20.4(6.2)^{a-c, e}$ \\
\hline Glucose (mM) & $5.7(1.4)^{f}$ & $5.8(1.3)^{f}$ & $5.7(1.4)^{f}$ & $6.0(1.3)^{f}$ & $5.8(1.1)^{f}$ & $6.3(1.9)^{a-e}$ \\
\hline $\mathrm{HDL}(\mathrm{mM})$ & $1.3(0.4)^{\mathrm{cdf}}$ & $1.4(0.4)^{\mathrm{cdf}}$ & $1.2(0.3)^{\mathrm{ab}}$ & $1.2(0.3)^{\mathrm{ab}}$ & $1.3(0.3)$ & $1.2(0.3)^{\mathrm{ab}}$ \\
\hline Triglycerides (mM) & $1.4(0.9)^{\mathrm{df}}$ & $1.5(0.9)^{f}$ & $1.4(0.9)^{f}$ & $1.7(0.8)^{\mathrm{a}}$ & $1.5(0.9)$ & $1.8(1.0)^{\mathrm{a}-\mathrm{c}}$ \\
\hline $\mathrm{SBP}(\mathrm{mmHg})$ & $125(12)^{\mathrm{df}}$ & $128(14)^{\text {af }}$ & $125(13)^{\mathrm{df}}$ & $131(13)^{a, c, f}$ & $128(12)^{f}$ & $135(14)^{a-e}$ \\
\hline $\mathrm{DBP}(\mathrm{mmHg})$ & $78(7)^{\mathrm{df}}$ & $79(8)^{f}$ & $78(7)^{\mathrm{df}}$ & $80(10)^{\text {acf }}$ & $81(10)^{f}$ & $83(9)^{a-e}$ \\
\hline T2D Med (\%) & $12.1^{\mathrm{df}}$ & 18.5 & 18.3 & $23.2^{\mathrm{a}}$ & 18.4 & $25.9^{\mathrm{ae}}$ \\
\hline BP Med (\%) & $31.8^{\mathrm{d}, \mathrm{f}}$ & $40.4^{\mathrm{d}, \mathrm{f}}$ & $28.3^{\mathrm{d}, \mathrm{f}}$ & $55.8^{\mathrm{a}-\mathrm{c}, \mathrm{e}}$ & $23.7^{d, f}$ & $55.1^{\mathrm{a}-\mathrm{c}, \mathrm{e}}$ \\
\hline Lipid Med (\%) & 30.8 & $37.1^{c}$ & $21.7^{\mathrm{bdf}}$ & $40.9^{c, e}$ & $21.1^{\mathrm{df}}$ & $37.7^{c, e}$ \\
\hline
\end{tabular}

Mild Obesity (BMI: $30-34.9 \mathrm{~kg} / \mathrm{m}^{2}$ ); Moderate obesity (BMl: $35-39.9 \mathrm{~kg} / \mathrm{m}^{2}$ ); Severe obesity (BMI: $\geq 40 \mathrm{~kg} / \mathrm{m}^{2}$ ). Fit: top 80 th percentile for age and sex categories $B M I$ body mass index, $V_{2 \max }$ maximal oxygen consumption, $H D L$ high density lipoprotein, SBP systolic blood pressure, DBP diastolic blood pressure, $T 2 D$ type 2 diabetes ${ }^{a}$ Significantly different compared to fit-mild obesity $(P<0.05)$

${ }^{\mathrm{b}}$ Significantly different compared to unfit-mild obesity $(P<0.05)$

'Significantly different compared to fit-moderate obesity $(P<0.05)$

${ }^{\mathrm{d}}$ Significantly different compared to unfit-moderate obesity $(P<0.05)$

e'Significantly different compared to fit-severe obesity $(P<0.05)$

f Significantly different compared to unfit-severe obesity $(P<0.05)$

difference between fitness groups within a given BMI category was in the relative risk for prevalent pre-clinical hypertension within the severe obesity group (Fit versus Unfit RR: 0.77, 0.59-0.99, $p=0.047$ ).

\section{Discussion}

To our knowledge, this is the first study to demonstrate that the association between fitness and health may be similar if not augmented in individuals with severe obesity as compared to mild obesity and extends previous research done in populations with normal weight, overweight and mild obesity. Further, we suggest that these differences in health risk by fitness may be related with differences in waist circumference.

High fitness is commonly defined in the health literature as being in the top $80 \%$ of an age- and sex-category as this is the threshold often reported as being associated with the greatest gains in health benefits $[1,2]$. This means that the $10 \%$ prevalence of high fitness observed in those with severe obesity is 8 times lower than what would be expected in the general population. Within the present study, fitness was predicted using symptom-limited treadmill testing. Therefore, some individuals may have stopped due to problems such as musculoskeletal pain which is shown to be more likely in populations with obesity [16] rather than cardiovascular fatigue. Also, certain medications that are more prevalent in populations with obesity, such as beta blockers, can influence heart rate and blood pressure and increase the likelihood of experiencing early cardiovascular fatigue during exercise [17]. Given that our sample comprised individuals with higher levels of obesity who are likely to have more health problems, our ability to accurately identify patients with high fitness may have been reduced. Nevertheless, the concept that an individual can present with high fitness despite severe obesity is a novel observation that has important clinical and public health implications.

Several studies have demonstrated a positive association between obesity and metabolic risk factors, as well as an inverse association between fitness and metabolic risk [2, 3]. These observations are largely limited to populations consisting of mainly normal weight, overweight and mild obesity [1,2]. Borodulin et al. [8] and Lee et al. [6] demonstrated that there was a stronger association between CRF and systolic blood pressure with increasing levels of adiposity. Conversely, data from the Aerobics Centre Longitudinal Study suggest that the association between fitness and blood pressure may be weaker in those with greater obesity [18]. We extend these findings to demonstrate that the benefits of fitness for most of the metabolic risk factors are similar for all obesity classes. The lone exception was that those with severe obesity, individuals who were fit were less likely to have pre-clinical or clinical hypertension than those were unfit. 

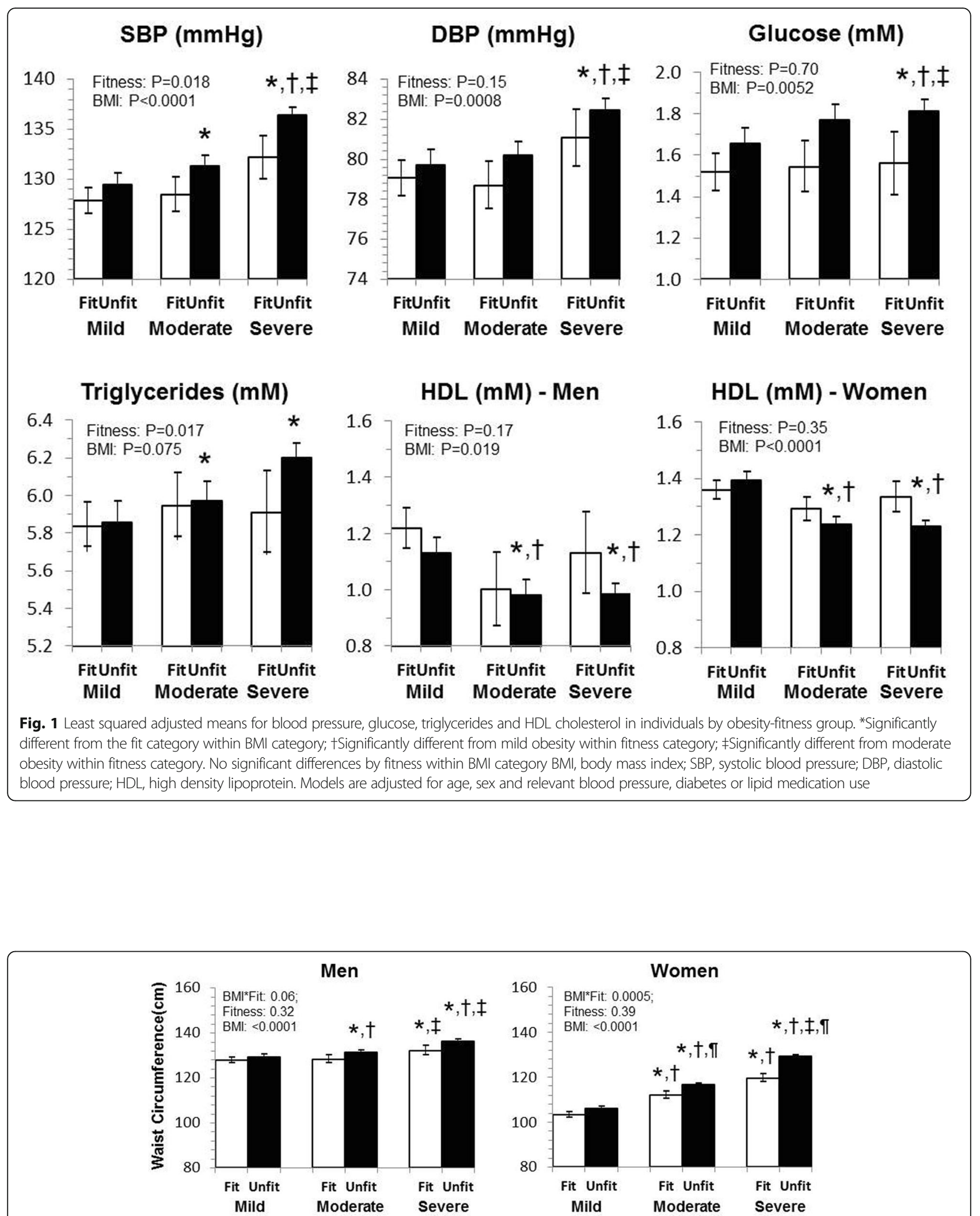

Fig. 2 Least squared adjusted means for waist circumference in men and women by obesity-fitness group. *Significantly different from the fit category within BMI category; +Significantly different from mild obesity within fitness category; \#Significantly different from moderate obesity within fitness category. ISignificantly different from the fit category within BMl category Models are adjusted for age 

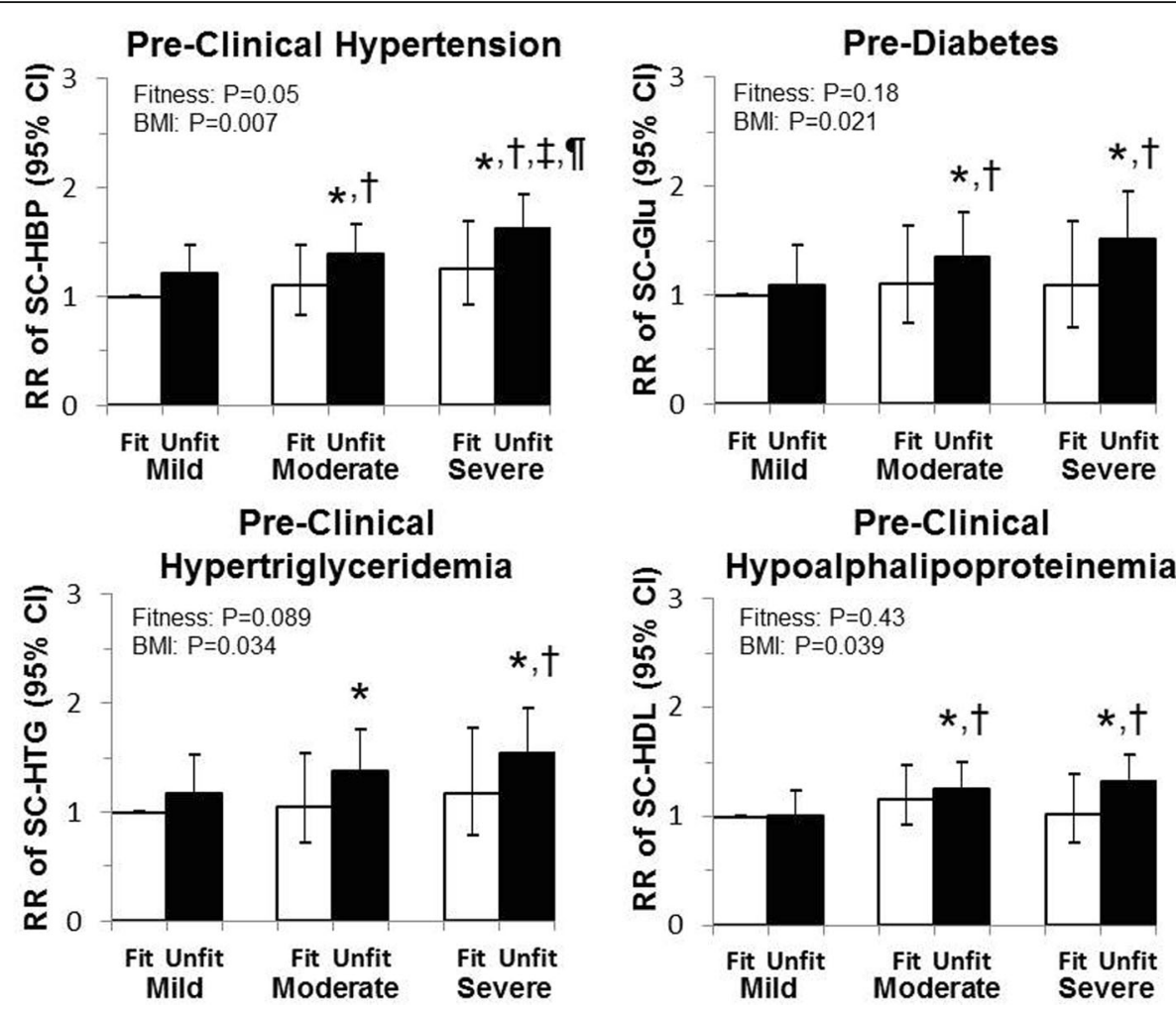

Fig. 3 Relative risk for preclinical hypertension, diabetes and hypertriglyceridemia and hypoalphalipoproteinemia in men and women by obesityfitness group. *Significantly different from the fit category within BMI category; +Significantly different from mild obesity within fitness category; ¥Significantly different from moderate obesity within fitness category. $\mathbf{9}$ Significantly different from the fit category within BMI category. Models are adjusted for age and sex

The health effects of fitness are suggested to be mediated in part through the positive health benefits of engaging in regular physical activity [2]. Physical activity has been shown to improve fasting glucose by increasing the rate of glucose uptake in skeletal muscle [19] and improve lipid metabolism through increases in lipoprotein lipase in both skeletal muscle and adipose tissue [20]. As individuals with severe obesity are more likely to have deteriorations in these metabolic risk factors, it may not be surprising that the benefits of fitness may also extend to those with severe obesity. Nevertheless, in our study and others [18, 21, 22], obesity was more strongly associated with metabolic health risk than fitness. However, it should be of note that within the fit individuals, those with severe obesity did not have significantly elevated glucose, blood pressure or lipids as compared to those with mild obesity. This supports the potentially important health benefits of having a high fitness level, particularly for those with severe obesity.

The beneficial effects of fitness on health may also be attributed to differences in abdominal obesity. Wong et al. [5] report that in men the differences in visceral obesity with high fitness are reduced with increasing BMI and may be completely abolished at a BMI of $\geq 35 \mathrm{~kg} / \mathrm{m}^{2}$. This would suggest that the benefits of fitness should be attenuated with those with moderate and severe obesity. However, our results in women and to a lesser degree in men, demonstrate that there was a greater difference in waist circumference between fitness groups in the moderate and severe obesity group than the mild obesity group: an observation that mirrors our results for metabolic health. These differences may in part steam from the sex or other demographic differences between the ACLS and WMC populations studied, but may suggest that fitness may be particularly important for women with obesity. However, these primary observations need further investigation for confirmation.

The strengths and limitations of our study warrant mention. Although this study used a larger sample size with higher levels of obesity than previous studies, the cross sectional design of our study does not allow us to infer causality. Also, we were unable to adjust for other factors such as physical activity, ethnicity, smoking status, and education as these variables were not consistently reported within our clinical population.

\section{Conclusions}

With the increasing prevalence of obesity [23], efforts to understand variation in health risk within this 
population are of considerable public health importance. We demonstrate that the benefits of fitness on metabolic health appear similar if not augmented in those with higher levels of obesity as compared to those with lower levels of obesity. Thus, it may be equally if not more important to promote physical activity and fitness behaviours to this increasingly prevalent group of individuals in order to obtain metabolic health benefits.

\section{Acknowledgements}

This work was supported by the Canadian Institute of Health Grant no. 131594 We thank Sarah Vanderlelie and Rebecca Christensen for their dedication and administrative support to the ongoing research at WMC.

\section{Funding}

JLK and CIA received funding from CIHR. KD received graduate student funding from the ClHR grant. CIHR had no role in the design of the study and collection analysis, and interpretation of data and in writing the manuscript. SW is the founder and employee of WMC and contributed as an author to the study.

\section{Availability of data and materials}

The datasets generated and analysed during the current study are not publicly available due to privacy laws associated with medical data but are available with a data sharing agreement as approved by the relevant institutional ethics committee and the health information custodian (S. Wharton).

\section{Authors' contributions}

$\mathrm{KD}, \mathrm{REB}, \mathrm{SW}, \mathrm{ClA}$ and JLK made substantial contributions to conception and design. $J K$ and MR were responsible for the analysis and interpretation of data. KD, REB, SW, CIA and JLK were involved in revising the manuscript critically for important intellectual content; and gave final approval of the version to be published. Each author takes responsibility for the content; and agrees to be accountable for all aspects of the work in ensuring that questions related to the accuracy or integrity of any part of the work are appropriately investigated and resolved.

\section{Ethics approval and consent to participate}

All participants provided written informed consent knowing their decision to participate would not alter the care provided and that they could withdraw consent at any time. York University Institutional Review Board approved the study protocol used (Certificates: 2013-123 and \#e2017-166).

\section{Consent for publication}

Not applicable

\section{Competing interests}

SW is the founder and employee of WMC. The other authors have no competing interests.

\section{Publisher's Note}

Springer Nature remains neutral with regard to jurisdictional claims in published maps and institutional affiliations.

\section{Author details}

${ }^{1}$ School of Kinesiology and Health Science, York University, School of Kinesiology and Health Science, Toronto M3J 1P3, Canada. ${ }^{2}$ The Wharton Medical Clinic, Toronto, ON, Canada.

Received: 19 October 2017 Accepted: 23 January 2018

Published online: 31 January 2018

\section{References}

1. Lee CD, Blair SN, Jackson AS. Cardiorespiratory fitness, body composition, and all-cause and cardiovascular disease mortality in men. Am J Clin Nutr. 1999:69:373-80

2. Ortega FB, Lavie CJ, Blair SN. Obesity and cardiovascular disease. Circ Res. 2016;118:1752-70
3. Lavie CJ, De Schutter A, Parto P, Jahangir E, Kokkinos P, Ortega FB, Arena R, Milani RV. Obesity and prevalence of cardiovascular diseases and prognosisthe obesity paradox updated. Prog Cardiovasc Dis. 2016;58:537-47.

4. Oktay AA, Lavie CJ, Kokkinos PF, Parto P, Pandey A, Ventura HO. The interaction of Cardiorespiratory fitness with obesity and the obesity paradox in cardiovascular disease. Prog Cardiovasc Dis. 2017:60:30-44.

5. McAuley PA, Blaha MJ, Keteyian SJ, Brawner CA, Al Rifai M, Dardari ZA, Ehrman JK, Al-Mallah MH. Fitness, fatness, and mortality: the FIT (Henry ford exercise testing) project. Am J Med. 2016;129:960-965.e961.

6. Lee S, Kuk JL, Katzmarzyk PT, Blair SN, Church TS, Ross R. Cardiorespiratory fitness attenuates metabolic risk independent of abdominal subcutaneous and visceral fat in men. Diabetes Care. 2005;28:895-901.

7. Wong SL, Katzmarzyk P, Nichaman MZ, Church TS, Blair SN, Ross R. Cardiorespiratory fitness is associated with lower abdominal fat independent of body mass index. Med Sci Sports Exerc. 2004;36:286-91.

8. Borodulin K, Laatikainen T, Lahti-Koski M, Lakka TA, Laukkanen R, Sarna S, Jousilahti P. Associations between estimated aerobic fitness and cardiovascular risk factors in adults with different levels of abdominal obesity. Eur J Cardiovasc Prev Rehabil. 2005;12:126-31.

9. Twells LK, Gregory DM, Reddigan J, Midodzi WK. Current and predicted prevalence of obesity in Canada: a trend analysis. CMAJ Open. 2014;2:E18-26.

10. Lohman TG, Roche AF, Martello R. Anthropometric standardization reference manual. Champaign, IL: Human Kinetics; 1988.

11. Lau DC, Douketis JD, Morrison KM, Hramiak IM, Sharma AM, Ur E. 2006 Canadian clinical practice guidelines on the management and prevention of obesity in adults and children. CMAJ. 2007:176:S1-117.

12. Pollock ML, Foster C, Schmidt D, Hellman C, Linnerud AC, Ward A. Comparative analysis of physiologic responses to three different maximal graded exercise test protocols in healthy women. Am Heart J. 1982;103:363-73.

13. Balke B, Ware RW. An experimental study of physical fitness in air force personnel. US Armed Forces Med J. 1959;10:675-88.

14. Blair SN, Kohl HW 3rd, Paffenbarger RS Jr, Clark DG, Cooper KH, Gibbons LW Physical fitness and all-cause mortality. A prospective study of healthy men and women. JAMA. 1989;262:2395-401.

15. Zou G. A modified poisson regression approach to prospective studies with binary data. Am J Epidemiol. 2004;159:702-6.

16. Hulens M, Vansant G, Lysens R, Claessens AL, Muls E. Exercise capacity in lean versus obese women. Scand J Med Sci Sports. 2001;11:305-9.

17. Noonan V, Dean E. Submaximal exercise testing: clinical application and interpretation. Phys Ther. 2000;80:782-807.

18. Chen J, Das S, Barlow CE, Grundy S, Lakoski SG. Fitness, fatness, and systolic blood pressure: data from the Cooper Center longitudinal study. Am Heart J. 2010;160:166-70

19. O'Gorman DJ, Karlsson HK, McQuaid S, Yousif O, Rahman Y, Gasparro D, Glund S, Chibalin AV, Zierath JR, Nolan JJ. Exercise training increases insulinstimulated glucose disposal and GLUT4 (SLC2A4) protein content in patients with type 2 diabetes. Diabetologia. 2006;49:2983-92.

20. Nikkila EA, Taskinen MR, Rehunen S, Harkonen M. Lipoprotein lipase activity in adipose tissue and skeletal muscle of runners: relation to serum lipoproteins. Metab Clin Exp. 1978:27:1661-7.

21. Lakoski SG, Barlow CE, Farrell SW, Berry JD, Morrow JR Jr, Haskell WL. Impact of body mass index, physical activity, and other clinical factors on cardiorespiratory fitness (from the Cooper Center longitudinal study). Am J Cardiol. 2011;108:34-9.

22. Weinstein AR, Sesso HD, Lee IM, Cook NR, Manson JE, Buring JE, Gaziano JM. Relationship of physical activity vs body mass index with type 2 diabetes in women. JAMA. 2004:292:1188-94.

23. Flegal KM, Carroll MD, Ogden $\mathrm{CL}$, Johnson $\mathrm{CL}$. Prevalence and trends in obesity among US adults, 1999-2000. JAMA. 2002;288:1723-7. 